\title{
Ziv-aflibercept in metastatic colorectal cancer
}

\author{
This article was published in the following Dove Press journal: \\ Biologics:Targets and Therapy \\ 13 December 2013 \\ Number of times this article has been viewed
}

\author{
Anuj Patel \\ Weijing Sun \\ Division of Hematology-Oncology, \\ University of Pittsburgh Cancer \\ Institute, University of Pittsburgh \\ School of Medicine, Pittsburgh, PA, \\ USA
}

Correspondence: Weijing Sun Division of Hematology-Oncology, University of Pittsburgh Cancer Institute, UPMC Cancer Pavilion, 5150 Centre Avenue, Fifth Floor, Pittsburgh, PA 15232, USA

Tel + I 4I2864 7764

Fax + I 4126486579

Email sunw@upmc.edu
Abstract: The combination of cytotoxic chemotherapy and antiangiogenic agents has become a conventional treatment option for patients with metastatic colorectal cancer. Ziv-aflibercept is a fusion protein which acts as a decoy receptor for vascular endothelial growth factor (VEGF)-A, VEGF-B, and placental growth factor (PlGF); it was approved in combination with 5-fluorouracil, leucovorin, and irinotecan (FOLFIRI) for the treatment of patients with metastatic colorectal cancer that is resistant to or has progressed after an oxaliplatin-containing fluoropyrimidine-based regimen. Herein we review the role of tumor angiogenesis as the rationale for antiangiogenic therapy, the clinical data associated with ziv-aflibercept, and its current role as a treatment option compared to other antiangiogenic agents, such as bevacizumab and regorafenib.

Keywords: aflibercept, angiogenesis, colorectal cancer

\section{Introduction}

Colorectal cancer (CRC) is the fourth most common cause of death from cancer worldwide, accounting for approximately $8 \%$ of all cancer deaths. ${ }^{1}$ Nearly $25 \%$ of patients will present with distant metastases; up to half of those with earlier-stage presentations will eventually progress to metastatic disease. ${ }^{2}$ These patients currently carry a poor prognosis, with a 5-year survival rate of approximately $10 \%{ }^{3}$ Recent research in metastatic CRC ( $\mathrm{mCRC}$ ) has focused primarily on the addition of biological target-oriented therapies to combination cytotoxic chemotherapy regimens. The monoclonal antibody bevacizumab, which binds and blocks the proangiogenic factor vascular endothelial growth factor (VEGF)-A, was the first successful targeted therapy in $\mathrm{mCRC}$ and the first antiangiogenic agent approved for use in cancer. The incorporation of antiangiogenic agents into the treatment of $\mathrm{mCRC}$ has led to improved antitumor responses and increased survival. Ziv-aflibercept is a recombinant inhibitor of the VEGF pathway. This article aims to review the data supporting its use in mCRC and its role in current practice. It will also provide an overview of tumor angiogenesis, with a focus on the VEGF signaling pathway.

\section{Tumor angiogenesis}

Angiogenesis, the formation of new blood vessels from existing vessels, is an essential process in tissue development and growth. These blood vessels facilitate the delivery of necessary oxygen and nutrients, as well as the removal of waste products. Angiogenesis is normally finely coordinated through the balance and interaction of proangiogenic and antiangiogenic signaling pathways. ${ }^{4}$ Proangiogenic factors such as 
VEGF are appropriately upregulated in a number of physiologic settings, including embryogenesis, bone formation, wound healing, and ovarian follicle growth. Dysregulation of angiogenesis plays a key role in certain diseases, such as arthritis, psoriasis, macular degeneration, diabetic retinopathy, and cancer. ${ }^{5}$

Pathologic angiogenesis is a key component of cancer growth ${ }^{6}$ and a necessary process for tumor metastasis. ${ }^{7}$ Tumor growth beyond 2-3 $\mathrm{mm}$ has been shown to be dependent on angiogenesis. ${ }^{8}$ An angiogenic switch has been described as a discrete step in the progression of tumors, required before they develop the potential for increased size, invasion, and metastasis. This switch can involve the overexpression of proangiogenic factors and increased sensitivity to these factors. ${ }^{9}$

In the majority of cancers, tumor angiogenesis proceeds by promoting vessel dilation and perivascular detachment in normal vessels in the vicinity of the tumor. This leaves them susceptible to the migration of rapidly recruited endothelial cells necessary for sprouting angiogenesis and the formation of new blood vessels branching off from existing vessels., ${ }^{9,10}$ Some tumors, such as astrocytomas, instead progress initially by growing along and co-opting normal blood vessels. As these tumors enlarge beyond the coverage of these co-opted vessels, they develop regions of hypoxia and necrosis. Factors triggered by these conditions, in turn, promote sprouting angiogenesis to support continued tumor growth. ${ }^{9,11}$ The blood vessels formed in tumors are irregular and tortuous. They form chaotically, without the hierarchy seen in normal vessels. This network is often leaky and predisposed to hemorrhage. ${ }^{9}$ Dysfunctional blood flow in these vessels may result in further ischemia, driving additional angiogenesis and leading to increased microvessel density. Intratumor microvessel density has been proposed as a prognostic factor in a number of cancers. ${ }^{12}$ The permeable vasculature may also provide accessibility for tumor cell invasion as a step in metastasis. ${ }^{13}$

\section{The VEGF pathway}

The VEGF signaling pathway is the best understood angiogenic pathway. The VEGF ligand family in humans consists of 5 glycoproteins, VEGF-A, VEGF-B, VEGF-C, VEGF-D, and placental growth factor (PlGF). These bind to three separate receptor tyrosine kinases (RTKs), VEGF receptor (VEGFR)-1 (FLT-1), VEGFR-2 (KDR/FLK-1), and VEGFR-3 (FLT-4), which are found primarily on the surface of vascular and lymphatic endothelial cells. Two neuropilins, NRP-1 and NRP-2, act as coreceptors for
VEGFRs, increasing the binding affinity of their respective ligands. ${ }^{14}$

The role of VEGF-A, also known simply as VEGF or as vascular permeability factor (VPF), has been the most closely explored. VEGF-A binds to both VEGFR-1 and VEGFR-2. The VEGF-A/VEGFR-2 pathway is thought to be the dominant promoter of angiogenesis. ${ }^{15,16}$ The activation of VEGFR-2 triggers multiple intracellular signaling cascades, including those that promote endothelial cell proliferation, migration, survival, and the increase in vessel permeability necessary for new microvessel formation. VEGF-A also promotes mobilization of the necessary endothelial cell precursors from the bone marrow. ${ }^{17,18}$ Numerous processes modulate the expression and activity of VEGF-A. Several growth factors, such as transforming growth factor (TGF) $\alpha$, TGF $\beta$, basic fibroblast growth factor (bFGF), keratinocyte growth factor, insulin-like growth factor-1, and plateletderived growth factor have been shown to promote VEGF-A messenger ribonucleic acid (mRNA) expression. ${ }^{5} \mathrm{VEGF}-\mathrm{A}$ expression is also upregulated by several oncogenic mutations, such as those in p53, RAS, SRC, and human epithelial growth factor receptor (HER)2. Oxygen tension also plays a key role in the regulation of VEGF-A. Hypoxia, a prevalent component of tumor growth, increases VEGF-A expression through the activation of hypoxia-inducible factors (HIF) HIF- $1 \alpha$ and HIF-2 $\alpha .^{13,19,20}$ Increased VEGF-A levels are seen in almost all malignancies, ${ }^{21,22}$ and increased expression is often found within the tumor cells themselves. The majority of tumor cells do not express VEGFR-2; VEGFR-2 expression, however, is 3-5-fold higher in the vasculature of tumors than in normal tissue. ${ }^{23}$ This pattern supports a paracrine signaling loop between VEGF-A and VEGFR-2 driving tumor angiogenesis.

The functions of VEGFR-1 with its respective ligands are less clearly understood. In addition to VEGF-A, VEGF-B and PlGF also bind with VEGFR-1. There is evidence that VEGFR-1 can act on angiogenesis in either a stimulatory or inhibitory fashion, depending on which ligand is bound. ${ }^{14,24,25}$ VEGFR-1-knockout mice die in utero due to excess proliferation and disorganization of blood vessels. By comparison, mice with a variant VEGFR-1, able to bind its ligands but lacking tyrosine kinase (TK) activity, do not show vascular defects. These findings highlight the critical negative regulatory role of VEGFR-1 during embryogenesis and support a mechanism of action as a non-signaling decoy receptor, binding and sequestering VEGF-A. ${ }^{26}$ VEGFR-1 binds VEGF-A with almost an order of magnitude higher affinity than VEGFR-2; 
however, it has a 10 -fold weaker tyrosine kinase activity than VEGFR-2. ${ }^{24}$

There is similar evidence of protumor activity of VEGFR-1; the mechanisms of this activity are still unexplained. In a model of human colorectal cancer in mice, ribozyme disruption of either VEGFR-1 or VEGFR-2 reduced tumor growth and metastasis. In a lung cancer model, both ribozymes inhibited primary tumor growth of lung cancer; however, only disruption of VEGFR-1 resulted in decreased pulmonary metastases. ${ }^{27}$ Similarly, pulmonary metastases from lung cancer xenografts were increased in those VEGFR-1-TK-deficient mice. ${ }^{24}$ VEGFR-1 is upregulated in a number of solid tumors and VEGFR-1-positivity predicts a poor prognosis, including recurrence and metastasis, in non-small-cell lung cancer and breast cancer. ${ }^{26}$

VEGFR-1 may play a more proangiogenic, protumor role in the presence of pathologically increased ligands. Xenografts of human PlGF-overexpressing murine lung cancer cells grow faster in VEGFR-1 wild-type mice than in VEGFR-1-TK-deficient mice. ${ }^{28} \mathrm{PlGF}$ has been shown to promote angiogenesis in in vivo models. ${ }^{25}$ Loss of PlGF impairs angiogenesis in ischemia, wound healing, and in tumors. However, embryonic angiogenesis in PlGF-deficient mice is unaffected and these mice remain healthy and viable. ${ }^{29}$ In normal conditions, PlGF expression is undetectable in most tissue, but it is upregulated in numerous pathological conditions.$^{26}$ These findings suggest that PlGF plays a role in angiogenesis primarily in pathologic conditions only. PlGF levels are increased in a number of cancers and associated with stage, metastasis, and survival in certain malignancies. ${ }^{26}$ Under certain settings, however, PlGF also appears to play an inhibitory role in tumor angiogenesis. In one study, lung, colon, and glioblastoma tumors transfected with PIGF grew slower in vivo than the same tumors without. Microvessel density was also decreased within the transfected tumors. ${ }^{30}$ This range from proangiogenic to antiangiogenic activity can be explained, in part, by the varied interactions between PlGF and VEGF-A. PIGF can promote VEGF-A activity by binding to VEGFR-1 and preventing sequestration of VEGF-A. PlGF/ VEGFR-1 binding also appears to lead to transphosphorylation of VEGFR-2, markedly increasing angiogenic activity. ${ }^{31}$ And, though notably weaker than VEGF-A/VEGFR-2 activity, PlGF does stimulate angiogenesis directly when binding to VEGFR-1. This proangiogenic relationship is generally prevalent when VEGF-A and PIGF are expressed by separate cells. Both factors, however, can be expressed simultaneously in the same cell. ${ }^{32}$ In this setting, VEGF-A and PIGF can form heterodimers, which demonstrate weaker angiogenic activity than VEGF-A homodimers. VEGF-A/PIGF heterodimers were observed in increased levels in the aforementioned PIGF transfected tumors. ${ }^{31,32}$

Like PlGF, VEGF-B appears to be largely dispensable during normal embryogenesis and development. VEGF-Bdeficient mice are generally viable and healthy through adulthood, though they do develop minor cardiac abnormalities..$^{33}$ This apparent role in cardiac development corresponds with the increased expression of VEGF-B seen in embryonic and adult myocardium. ${ }^{31}$ VEGF-B binding to VEGFR-1 does not appear to efficiently stimulate angiogenesis. ${ }^{25}$ It has, however, been seen to facilitate cell survival in normal vasculature. ${ }^{34}$ Overexpression of VEGF-B is found in a number of solid tumor malignancies, where it is often associated with disease stage. ${ }^{26}$ Immunohistochemical evaluation of tumor tissue from CRC patients showed VEGF-B positivity in the intratumor vessels of $>80 \%$ of samples; however, most of this was seen on preexisting, not angiogenic vessels. VEGF-B was significantly correlated with hematogenous metastases. ${ }^{35}$ These findings suggest that the role of VEGF-B in tumor progression is centered more on maintaining existing vasculature than on driving angiogenesis.

VEGF-C and VEGF-D bind to both VEGFR-2 and VEGFR-3. In adults, VEGFR-3 is expressed primarily on the lymphatic endothelium. Both VEGF-C and VEGF-D have been noted to promote lymphangiogenesis and lymph node metastasis through their binding with VEGFR-3. ${ }^{36,37}$ This appears to be the primary role of these ligands; however, both can also promote angiogenesis through their interaction with VEGFR-2. ${ }^{36,38}$ VEGF-C levels have been associated with poor prognosis in a number of cancers, including non-small-cell lung cancer and CRC. . $^{37,39,40}$

The VEGF pathways intersect with a number of other signaling pathways involved in the coordination of tumor angiogenesis. The angiopoietins (Ang-1, Ang-2, and Ang-4) interact with the RTKs tyrosine kinase with immunoglobulinlike and EGF-like domains (Tie)1 and Tie2. Both Ang-1 and Ang-2 have been associated with protumor and antitumor angiogenic effects. ${ }^{41}$ Ang-2, found on endothelial cells, is felt to destabilize vessels and amplify the activity of VEGF-A. ${ }^{13}$ Overexpression of Ang-2 and higher Ang-2:Ang-1 ratios were found to be correlated with poor prognosis in many cancers, including mCRC. ${ }^{41}$ Integrins expressed on varied cell surfaces also promote angiogenesis and interact with both the VEGF and Ang-Tie pathways. ${ }^{20}$

The Notch-signaling pathway consists of the ligands Jagged1, Jagged2, delta-like ligand (DLL) 1, DLL3, DLL4, and the Notch receptors, Notch1-4. DLL4 is upregulated 
by VEGF-A, and is felt to represent a negative feedback mechanism to angiogenesis under physiologic conditions. ${ }^{16}$ In tumor cells, however, the interaction between DLL4expressing and Notch1-expressing cells has been observed as a component of sprouting angiogenesis. ${ }^{41}$ Notch signaling has been shown to play a critical role in multiple cancers, including CRC. ${ }^{42}$ Increased DLL4 expression was found preferentially in CRC endothelial cells and was associated with VEGF expression. ${ }^{43}$ Notch signaling has also been shown to promote angiogenesis via upregulation of VEGFR-3. This angiogenic activity proceeds independent of VEGF-A, VEGF-C, or VEGFR-2. ${ }^{44}$

\section{Antiangiogenesis in metastatic colorectal cancer}

VEGF signaling provides potential targets for antiangiogenic therapy in mCRC. Expression of the VEGF family members appears to be associated with the degree of angiogenesis and the biologic activity of CRC tumors. Of the VEGF ligands, VEGF-A is the most abundant in CRC cells. ${ }^{45}$ Increased levels of VEGF-A expression have been shown to be associated with tumor stage, lymph node and liver metastases, and overall survival. ${ }^{31,46,47}$ VEGF-C expression has been also associated with lymph node metastasis and poor prognosis in mCRC. ${ }^{37}$ Increased PlGF expression in CRC has also been associated with poor outcomes ${ }^{48}$ and PlGF levels may correlate independently with metastatic lymph node involvement, tumor stage, and patient survival. ${ }^{49}$ Correlations between VEGF-B and VEGF-D and clinical outcomes in CRC have been described, but these are inconsistent. ${ }^{47}$

Three antiangiogenic agents have been approved by the US Food and Drug Administration (FDA) for the treatment of mCRC: bevacizumab, ziv-aflibercept, and regorafenib. Bevacizumab is a recombinant humanized monoclonal antibody that binds to VEGF-A, preventing its binding to VEGFR-1 and VEGFR-2. Bevacizumab was approved for the first-line treatment of $\mathrm{mCRC}$ based on the results of a Phase III trial comparing irinotecan, bolus 5-FU, and leucovorin (IFL) plus bevacizumab to IFL alone in 813 patients with untreated mCRC. ${ }^{50}$ The addition of bevacizumab was associated with increased overall survival (OS) when compared to the control arm (median OS [mOS] 20.3 versus 15.6 months; hazard ratio $[\mathrm{HR}] 0.66 ; P<0.001$ ). Progression-free survival (PFS), response rate $(\mathrm{RR})$, and duration of response were all also significantly higher in the bevacizumab arm.

The subsequent E3200 study also demonstrated the benefit of bevacizumab in the second-line setting. ${ }^{51}$ Eight hundred and twenty-nine mCRC patients previously treated with fluoropyrimidine and irinotecan were randomized to one of three treatment arms: infusional 5-FU, leucovorin, oxaliplatin (FOLFOX4) and bevacizumab, FOLFOX4 alone, or bevacizumab alone. The mOS in the FOLFOX4-bevacizumab arm was 12.9 months, compared to 10.8 months for the FOLFOX4-alone arm (HR 0.75; $P=0.0011)$ versus 10.2 months for the bevacizumab-alone arm. Median PFS (mPFS) in the FOLFOX4-bevacizumab arm was 7.3 months, compared to 4.7 months for the FOLFOX4-alone (HR 0.61; $P<0.0001$ ) and 2.7 months in the bevacizumab-alone arm.

Early studies with bevacizumab also established a set of class-associated adverse effects attributed to anti-VEGF therapy. Hypertension was the most documented of these toxicities. The prevalence and degree of hypertension was dose-dependent and seen more frequently in studies utilizing bevacizumab at doses higher than $5 \mathrm{mg} / \mathrm{kg}$ every 2 weeks or $7.5 \mathrm{mg} / \mathrm{kg}$ every 3 weeks. ${ }^{52}$ Proteinuria was also seen in each study, which was generally mild and asymptomatic; rarely, it progressed to nephrotic syndrome or renal failure. Other associated adverse effects included impaired wound healing, hemorrhage, gastrointestinal perforation, arterial thromboembolism, and reversible posterior leukoencephalopathy syndrome. An association with venous thromboembolism (VTE) has been less well-defined. Pooled analysis and metaanalysis did not show a significant increase in VTE when evaluating prior bevacizumab studies. ${ }^{53}$

Ziv-aflibercept, also known as VEGF Trap, is a recombinant fusion protein composed of extracellular domains from both VEGFR-1 and VEGFR-2 fused to the Fc(a) region of human IgG1. ${ }^{54}$ It was designed to act as a decoy VEGF receptor, binding VEGF-A, VEGF-B, and PIGF, subsequently preventing their interactions with VEGFR-1 and VEGFR-2. The studies evaluating ziv-aflibercept in $\mathrm{mCRC}$ are discussed below.

Regorafenib is a small-molecule multikinase inhibitor with broad activity, inhibiting multiple oncogenic kinases (KIT, RET, RAF), platelet-derived growth factor receptor (PDGFR), fibroblast growth factor receptor (FGFR), and VEGFR-1-3.55,56 Regorafenib was approved for the treatment of mCRC patients previously treated with fluoropyrimidine, oxaliplatin, or irinotecan-based chemotherapy regimens, with prior anti-VEGF and/or anti-EGFR therapies. Regorafenib was evaluated in the Phase III CORRECT trial, which studied 760 patients with $\mathrm{mCRC}$ who had progressed during or within 3 months after the above therapies. ${ }^{56}$ The patients enrolled were heavily pretreated; $48.0 \%(n=365)$ had received four or more prior lines of systemic therapy for metastatic disease, and all had previously been treated with an 
anti-VEGF-A agent. Patients on the study were randomized to either regorafenib or placebo. Treatment with regorafenib was associated with a significant increase in survival, with a mOS of 6.4 months in the regorafenib arm versus 5.0 months in the placebo arm (HR 0.77; $P=0.052$ ). mPFS and disease control rates were also significantly higher in the regorafenib arm. The most common grade 3 or 4 adverse events increased in the regorafenib arm were hand-foot skin reaction, fatigue, diarrhea, hypertension, and rash.

\section{Ziv-aflibercept in colorectal cancer}

Ziv-aflibercept was designed to be a high-affinity blocker of VEGF-A, with a favorable pharmacokinetic and immunogenicity profile. ${ }^{54}$ It is a fully-human soluble decoy receptor protein engineered by fusing the second immunoglobulin (Ig) domain of human VEGFR-1 and the third Ig domain of human VEGFR-2 with the constant region $(\mathrm{Fc})$ of human IgG1.$^{57}$ In vitro assays of ziv-aflibercept in human umbilical vein endothelial cells demonstrated the ability to completely block the phosphorylation of VEGFR-2 by VEGF-A, thereby blocking VEGF-A-induced cell proliferation. The binding kinetics of ziv-aflibercept were compared to bevacizumab and another anti-VEGF-A antibody, ranibizumab. Ziv-aflibercept was found to bind to VEGF-A with approximately 100-fold higher affinity than either bevacizumab or ranibizumab. As expected, PlGF-2 was also bound with high affinity by zivaflibercept, but was not bound at all by either bevacizumab or ranibizumab. Ziv-aflibercept also showed markedly more potent blockade of VEGFR-1 or VEGFR-2 activation than either anti-VEGF-A antibody. ${ }^{58}$

A number of in vivo studies showed anti-tumor and anti-tumor vessel activity in a variety of tumor xenografts. As a single-agent, ziv-aflibercept led to decreases in tumor vessels and angiogenesis, tumor growth, and metastasis. Zivaflibercept also demonstrated synergy with other systemic treatments in a number of studies, leading to greater inhibition of tumor growth and change in tumor vasculature. In two studies, one of fibrosarcoma and one of glioblastoma, ziv-aflibercept also enhanced the effects of radiotherapy. ${ }^{59}$

\section{Phase I studies}

An initial Phase I dose-finding study investigated singleagent ziv-aflibercept in patients with advanced, refractory solid tumors or non-Hodgkin lymphoma. Forty-seven patients were given ziv-aflibercept intravenously every 2 weeks in a dose-escalating schema from 0.3 to $7.0 \mathrm{mg} / \mathrm{kg}$; seven of these patients had CRC. ${ }^{60}$ The treatment-related toxicities recorded were similar to those seen with other
anti-VEGF agents, including bevacizumab. These included hypertension, proteinuria, and dysphonia and were generally noted to be reversible with discontinuation of the drug. These toxicities were more prevalent and more severe at doses of $4.0 \mathrm{mg} / \mathrm{kg}$ and greater. Three subjects were found to have partial responses by response evaluation criteria in solid tumors (RECIST) criteria; none of these were colorectal cancer patients. Maximal levels of VEGF-bound ziv-aflibercept complex and of free ziv-aflibercept were found at doses $\geq 2.0 \mathrm{mg} / \mathrm{kg}$, indicating complete ligand blockade. On the basis of the toxicity and pharmacokinetic results, $4 \mathrm{mg} / \mathrm{kg}$ every 2 weeks was established as the recommended Phase II dose (RPTD) for future studies.

Several Phase I studies have evaluated ziv-aflibercept in combination with cytotoxic chemotherapy regimens; ${ }^{59}$ two of these focused primarily on patients with CRC. One dose-escalation trial studied ziv-aflibercept in combination with 5-fluorouracil, leucovorin, and irinotecan (FOLFIRI) in Japanese patients with $\mathrm{mCRC}$ who had undergone treatment with at least one prior chemotherapy regimen. ${ }^{61}$ Sixteen patients were treated with ziv-aflibercept and FOLFIRI; ziv-aflibercept was dosed at two levels: 2 and $4 \mathrm{mg} / \mathrm{kg}$. The PFS and RR at $4 \mathrm{mg} / \mathrm{kg}$ were 7.6 months and $7.8 \%$, respectively. No dose-limiting toxicities were seen at either dose level. The most common grade 3 or 4 adverse events were neutropenia and hypertension. Based on the efficacy and safety data, $4 \mathrm{mg} / \mathrm{kg}$ every 2 weeks was the RPTD of ziv-aflibercept in combination with FOLFIRI for Japanese patients with mCRC.

Another study evaluated patients with advanced solid tumors when treated with ziv-aflibercept and irinotecan, 5-flourouracil, and leucovorin (ILV5FU2). ${ }^{62}$ Of the 38 patients enrolled, 23 had CRC. Ziv-aflibercept was given in a doseescalating fashion at $2,4,5$, or $6 \mathrm{mg} / \mathrm{kg}$ intravenously on day 1 , followed by ILV5FU2 on days 1 and 2, cycled every 2 weeks. Nine patients had partial responses, 5 of these at $4 \mathrm{mg} / \mathrm{kg}$; 6 of the partial responses were seen in CRC patients. Dose limiting toxicities of proteinuria, nephrotic syndrome, stomatitis, febrile neutropenia were noted, starting with 2 patients at $4 \mathrm{mg} / \mathrm{kg}$. Based on the pharmacokinetic studies, response rates, and toxicity data, a dose of $4 \mathrm{mg} / \mathrm{kg}$ every 2 weeks was selected as the RPTD with ILV5FU2.

Following the dose-escalation stage of this study, a double-blind expansion stage randomized 27 new mCRC patients to ILV5FU2 and either ziv-aflibercept at $4 \mathrm{mg} / \mathrm{kg}$ every 2 weeks or placebo for cycle $1 .^{63}$ All patients received ziv-aflibercept with ILV5FU2 for subsequent cycles. Four patients achieved partial responses and 17 patients maintained 
stable disease. The most frequent grade 3 or 4 adverse events noted were neutropenia, fatigue, and hypertension; these were seen in $<40 \%$ of the patients treated.

\section{Phase II/III studies}

Three Phase II and III studies of ziv-aflibercept in mCRC have been completed (Table 1). The clinical activity of single-agent ziv-aflibercept was investigated in an open-label, two-stage, multicenter Phase II trial. ${ }^{64}$ Seventy-five patients with $\mathrm{mCRC}$, who had progressed on at least one prior line of systemic therapy, were enrolled into two separate cohorts based on prior bevacizumab exposure. The patient population accrued was heavily pretreated, with a median of two prior chemotherapy regimens with $81.3 \%(n=61)$ of patients previously treated with both oxaliplatin and irinotecan, and $46.7 \%(n=35)$ patients had prior exposure to an epidermal growth factor receptor (EGFR)-inhibitor. Seventy-four patients received ziv-aflibercept intravenously at a dose of $4 \mathrm{mg} / \mathrm{kg}$ every 2 weeks; 24 patients were enrolled in the bevacizumab-naïve cohort and 50 in the prior-bevacizumab cohort. The primary endpoint was a combination of objective response rate (ORR) and PFS at 16 weeks. No patients in the bevacizumab-naïve cohort achieved an objective response; five patients had stable disease at 16 weeks. In the priorbevacizumab cohort, one patient was found to have a partial response and six patients had stable disease at 16 weeks. mPFS in the bevacizumab-naïve cohort was 2.0 months versus 2.4 months in the prior-bevacizumab cohort. mOS was 10.4 months and 8.5 months, respectively. Without a randomized control arm, evaluation of these results was limited; however, when compared to the best supportive care arms of previous large clinical trials in $\mathrm{mCRC}$, this was not felt to represent meaningful single-agent activity. ${ }^{65,66}$ The most common grade 3 or 4 adverse events noted were hypertension, proteinuria, fatigue, and headache. Dose reduction of ziv-aflibercept was required in 12 patients and dosing was delayed due to toxicity in 20 patients. Ten patients discontinued study treatment due to adverse events.

The AFFIRM trial was a randomized, non-comparative Phase II trial designed to evaluate the use of ziv-aflibercept in the first-line treatment of mCRC.${ }^{67}$ Preliminary data from this study was presented at the European Society for Medical Oncology (ESMO) 14th World Congress on Gastrointestinal Cancer. Two hundred and thirty-six patients with previously untreated $\mathrm{mCRC}$ were randomized to receive either zivaflibercept ( $4 \mathrm{mg} / \mathrm{kg}$ every 2 weeks) and modified FOLFOX6

Table I Completed Phase II and III trials of ziv-aflibercept in metastatic colorectal cancer

\begin{tabular}{|c|c|c|c|}
\hline Trial & Patient population & Treatments & Results \\
\hline \multicolumn{4}{|l|}{ Phase II } \\
\hline PMH Phase II & Previously treated $\mathrm{mCRC}$ & Single-agent & Bevacizumab-naïve: \\
\hline Consortium ${ }^{64}$ & I+ prior lines of therapy & ziv-aflibercept & RR: $0 \%$ (0 PR, 5 SD at 16 weeks) \\
\hline \multirow[t]{6}{*}{ (NCT00407654) } & $(n=74)$ & & mPFS: 2.0 months (95\% Cl I.7-8.6) \\
\hline & & & mOS: 10.4 months $(95 \% \mathrm{Cl} 7.6-15.5)$ \\
\hline & Two cohorts: & & Prior-bevacizumab: \\
\hline & bevacizumab-naïve $(n=24)$ & & RR: $2.0 \%$ (I PR, 6 SD at I6 weeks) \\
\hline & prior-bevacizumab $(n=50)$ & & mPFS: 2.4 months (95\% Cl I.9-3.7) \\
\hline & & & mOS: 10.4 months $(95 \% \mathrm{Cl} 6.2-10.6)$ \\
\hline AFFIRM $^{67}$ & Treatment-naïve mCRC & mFOLFOX6 + ziv-aflibercept & RR: $49.1 \%$ (95\% Cl 39.7-58.6\%) \\
\hline \multirow[t]{6}{*}{ (NCT0085I084) } & no prior systemic treatment & $(n=\mid 19)^{\ddagger}$ & PFSI2: $25.8 \%$ (95\% Cl 17.2-34.4\%) \\
\hline & $(n=236)$ & & $\begin{array}{l}\text { mPFS: } 8.48 \text { months }(95 \% \mathrm{Cl} \\
7.89-9.92)\end{array}$ \\
\hline & & mFOLFOX6 $(n=117)^{\ddagger}$ & RR: $45.9 \%$ (95\% Cl 36.4-55.7\%) \\
\hline & & & PFSI2: $21.2 \%(95 \% \mathrm{Cl} 12.2-30.3 \%)$ \\
\hline & & & mPFS: 8.77 months $(95 \% \mathrm{Cl}$ \\
\hline & & & $7.62-9.27)$ \\
\hline \multicolumn{4}{|l|}{ Phase III } \\
\hline VELOUR ${ }^{68}$ & Previously treated mCRC & FOLFIRI + & RR: $19.8 \%$ versus $\mathrm{II} . \mathrm{I} \%(P<0.00 \mathrm{I})$ \\
\hline \multirow[t]{3}{*}{ (NCT0056/470) } & progression on or $\leq 6$ months & ziv-aflibercept & mPFS: 6.90 versus 4.67 months \\
\hline & after prior oxaliplatin-containing & $(\mathrm{n}=6 \mid 2)$ versus FOLFIRI + placebo & $(\mathrm{HR} 0.758 ; P<0.000 \mathrm{I})$ \\
\hline & regimen $(n=1,226)$ & $(n=6 \mid 4)$ & $\begin{array}{l}\text { mOS: } 13.50 \text { versus } 12.06 \text { months } \\
\text { (HR } 0.8 I 7 ; P=0.0032)\end{array}$ \\
\hline
\end{tabular}

Note: Study was non-comparative, not powered to compare two treatment arms.

Abbreviations: $\mathrm{Cl}$, confidence interval; FOLFIRI, 5-fluorouracil, leucovorin, and irinotecan; mCRC, metastatic colorectal cancer; HR, hazard ratio; metastatic colorectal cancer; mFOLFOX6, modified 5-fluorouracil, leucovorin, and oxaliplatin; mOS, median overall survivial; mPFS, median progression-free survival; NCT, National Clinical Trials; PFSI2, progression-free survival at 12 months; PMH, Princess Margaret Hospital; PR, partial response; RR, response rate; SD, stable disease. 
(mFOLFOX6; $\mathrm{n}=119)$ or mFOLFOX6 alone $(\mathrm{n}=117)$. While the patients enrolled were chemotherapy-naïve for metastatic disease, $97.5 \%$ had received prior adjuvant therapy. The mFOLFOX6 arm was included for calibration; the study was not powered to significantly compare the two arms. The primary endpoint of PFS at 12 months was $25.8 \%$ in the ziv-aflibercept arm and $21.2 \%$ in the mFOLFOX6-only arm, with mPFS of 8.48 months and 8.77 months, respectively. RR was $49.1 \%$ in the ziv-aflibercept arm and $45.9 \%$ in the mFOLFOX6-only arm. Grade 3-4 adverse events, including hypertension, proteinuria, neutropenia, diarrhea, and infections, were seen with $>5 \%$ higher incidence in the ziv-aflibercept arm.

\section{VELOUR trial}

Ziv-aflibercept was studied in combination with FOLFIRI in the Phase III VELOUR trial. ${ }^{68}$ This prospective, randomized, double-blind, multicenter, multinational study evaluated patients with $\mathrm{mCRC}$ previously treated with an oxaliplatinbased chemotherapy regimen. In all, 1,226 patients were randomized either to treatment with FOLFIRI plus zivaflibercept or FOLFIRI plus placebo. Patients were stratified by prior bevacizumab treatment and performance status; 853 patients (69.6\%) were bevacizumab-naïve and 373 patients $(30.4 \%)$ had prior exposure. With respect to metastatic disease, $89.6 \%(n=892)$ of patients had received treatment, while $10.4 \%(n=124)$ had been treated in an adjuvant setting only. In the experimental arm, ziv-aflibercept was dosed at $4 \mathrm{mg}$ / $\mathrm{kg}$ intravenously every 2 weeks, preceding FOLFIRI; placebo was also given intravenously prior to FOLFIRI.

The ziv-aflibercept arm showed significant improvement in the primary endpoint of OS, with mOS of 13.50 months compared to 12.06 months in the placebo arm (HR 0.817; $P=0.0032)$. mPFS and ORR were also increased; 6.90 months versus 4.67 months (HR 0.758; $P<0.0001)$ and 19.8\% versus $11.1 \%(P<0.001)$, respectively. In subgroup analyses of the prior-bevacizumab patients, PFS was significantly improved in both. OS was significantly improved in the bevacizumab-naïve subset, but did not achieve significance in the prior-bevacizumab subset. Based on the results of this study, the FDA approved the use of ziv-aflibercept in combination with FOLFIRI for the treatment of patients with $\mathrm{mCRC}$ that is resistant to or that has progressed after an oxaliplatin-containing regimen. Ziv-aflibercept was the first agent to demonstrate a survival benefit in this specific population.

Both antiangiogenic-associated and chemotherapyrelated adverse effects were seen with greater incidence in the ziv-aflibercept arm. Grade 3 or 4 adverse events were reported in $83.5 \%$ of patients in the ziv-aflibercept arm and $62.5 \%$ of patients in the placebo arm. The antiangiogenic toxicities described were hypertension, hemorrhage, and arterial and venous thromboembolism; all were higher in the ziv-aflibercept arm. Similarly, grade 3 or 4 chemotherapeutic adverse events, including diarrhea, asthenia, stomatitis, and myelosuppression were also found in higher incidence with ziv-aflibercept treatment. Adverse events led to discontinuation of treatment in $26.8 \%$ of patients in the ziv-aflibercept arm and $12.1 \%$ of patients in the control arm.

A prespecified subset analysis of elderly patients in VELOUR was presented at the ESMO 15 th World Congress on Gastrointestinal Cancer. ${ }^{69}$ Of the 1,226 patients randomized in the VELOUR study, 438 patients (36\%) were 65 years or older. An analysis of these patients revealed that OS was non-significantly increased with median OS of 12.6 months in the ziv-aflibercept arm and 11.3 months in the placebo arm (HR 0.853; 95\% confidence interval [CI] 0.682-1.066). PFS was also non-significantly greater, with a median PFS of 6.6 months and 4.4 months for the zivaflibercept and placebo arms, respectively (HR 0.748; 95\% CI 0.598-0.936). Dehydration was significantly associated with age $(P=0.0058)$; all other adverse effects were similar in proportion to the overall patient pool.

\section{Discussion}

Folkman first described tumor angiogenesis over four decades ago.$^{70}$ At that time, he postulated that antiangiogenic therapy could lead to decreased tumor growth and metastasis. The approval of bevacizumab for mCRC in 2004 marked the first approval of an antiangiogenic agent for the treatment of cancer. Since then, the landscape for antiangiogenic therapy in $\mathrm{CRC}$ has grown increasingly complex. In addition to the three FDA-approved drugs noted above, multiple newer agents targeting angiogenesis are currently under investigation in CRC. ${ }^{71,72}$ The role of ziv-aflibercept among these agents is unclear. No head-to-head clinical comparisons of ziv-aflibercept and bevacizumab, or other antiangiogenic agents, have been performed.

In a first-line setting, bevacizumab remains the only approved option for antiangiogenic therapy. Bevacizumab has been shown to improve survival in first-line treatment of mCRC in combination with IFL, ${ }^{50}$ FOLFIRI, ${ }^{73}$ and FOLFOX or XELOX (capecitabine [XELODA] and oxaliplatin $)^{74}$ and currently represents a standard-of-care consideration for appropriate patients. AFFIRM currently represents the only evaluation of ziv-aflibercept in first-line treatment. As shown 
above, the addition of ziv-aflibercept failed to show any clear benefit over cytotoxic chemotherapy (mFOLFOX6) alone; as noted, however, this study was not powered to demonstrate a significant difference.

Regorafenib is the only antiangiogenic agent evaluated as third-line or later treatment for mCRC. CORRECT showed a survival benefit with regorafenib in an extensively treated patient population, the majority of which had received three or more prior lines of systemic therapy. CORRECT also established regorafenib as the only antiangiogenic agent to date that has demonstrated a significant survival benefit as a single-agent in mCRC. Neither bevacizumab nor zivaflibercept showed evidence of single-agent benefit in earlier studies. ${ }^{51,64}$

\section{Second-line metastatic colorectal cancer therapy}

The results from VELOUR would seem to make an argument for incorporating ziv-aflibercept into second-line therapy, particularly in patients who had progressed through bevacizumab in the first-line. The more recently published results from the ML18147 (TML) trial have complicated this issue, however. This randomized Phase III trial addressed the role of bevacizumab continuation after disease progression. ${ }^{75}$ Eight hundred and twenty patients with $\mathrm{mCRC}$, who had progressed within 3 months of discontinuing first-line treatment with a bevacizumab-containing chemotherapy regimen, were randomized to second-line chemotherapy with or without bevacizumab. The choice of chemotherapy was determined by their prior treatment, with a change from irinotecan-based to oxaliplatin-based and vice versa. mOS improved to 11.1 months in the bevacizumab arm compared to 9.8 in the chemotherapy-only arm (HR $0.81 ; P=0.0062$ ); mPFS was also significantly increased (5.7 months versus 4.1 months; HR 0.68; $P<0.0001)$. RR was low (5\% versus $4 \%$ ) and not significantly different between the two arms. The FDA approved bevacizumab for its third indication in mCRC following the results of this trial (Table 2).

With these findings, there is currently no clear data to guide physicians when choosing between the addition of zivaflibercept or bevacizumab in the second-line setting. In the absence of a prospective, comparative trial, most comparisons have to be made by inference. The improvements in OS and PFS seen with the addition of ziv-aflibercept in VELOUR are roughly similar to those seen with bevacizumab in E3200 and ML18147. Of note, patients with prior bevacizumab exposure did have significantly improved OS in ML18147; as stated above, significance was not achieved in the prior- bevacizumab subset analysis of VELOUR. The grade 3 and 4 adverse events associated with ziv-aflibercept in VELOUR were also generally comparable to those with bevacizumab in earlier trials, with similar antiangiogenic-associated and chemotherapy-associated toxicities. ${ }^{76}$

Even prior to ML18147, questions arose regarding the costs and benefits of ziv-aflibercept versus bevacizumab. Without head-to-head comparison, discussions regarding this sensitive issue have become a part of the decisionmaking landscape. At the 2013 American Society of Clinical Oncology (ASCO) Gastrointestinal Cancers Symposium, a cost-effectiveness analysis of bevacizumab plus chemotherapy versus ziv-aflibercept plus chemotherapy in $\mathrm{mCRC}$ patients previously treated with bevacizumab was presented. ${ }^{77}$ Bevacizumab and ziv-aflibercept were compared through an adjusted indirect treatment comparison, using the hazard ratios from ML18147 and VELOUR, respectively. The results of the analysis favored bevacizumab. Likely affected by similar facts, Sanofi-Aventis began offering discounts of approximately $50 \%$ on the official price of the drug in November 2012.

\section{Anti-VEGF-A resistance}

The key to finding a role for ziv-aflibercept may lie in exploiting the unique aspects of its design and function. As described above, ziv-aflibercept does provide more potent binding of VEGFR-A and broader coverage of the VEGF pathway, with blockade of all the known ligands for VEGFR-1. One setting where this extended activity might prove beneficial is in patients who have developed resistance to pure VEGF-A inhibitors. Numerous resistance mechanisms to bypass VEGF-A-blockade have been proposed. In a Phase II trial of FOLFIRI and bevacizumab in patients with previously treated mCRC, plasma levels of VEGF-C, VEGF-D, PlGF, and basic fibroblast growth factor (bFGF), were significantly elevated before or at the time of disease progression. ${ }^{78}$ These factors have been associated with VEGF-A/VEGFR2-independent angiogenesis and have been implicated as potential mechanisms of anti-VEGF-A resistance. Increases in PlGF have been observed following anti-VEGF-A treatment in numerous other studies ${ }^{79,80}$ and anti-PlGF was shown to inhibit angiogenesis and tumor cell motility in mice with VEGF-A-inhibitor-resistant tumors. ${ }^{81}$ If we were able to select those tumors in which PlGF is the driving force behind anti-VEGF-A resistance, ziv-aflibercept might be considered as a rational second-line antiangiogenic agent.

Given that ziv-aflibercept did not show dramatic responses in the prior-bevacizumab cohort of VELOUR, it is likely that 


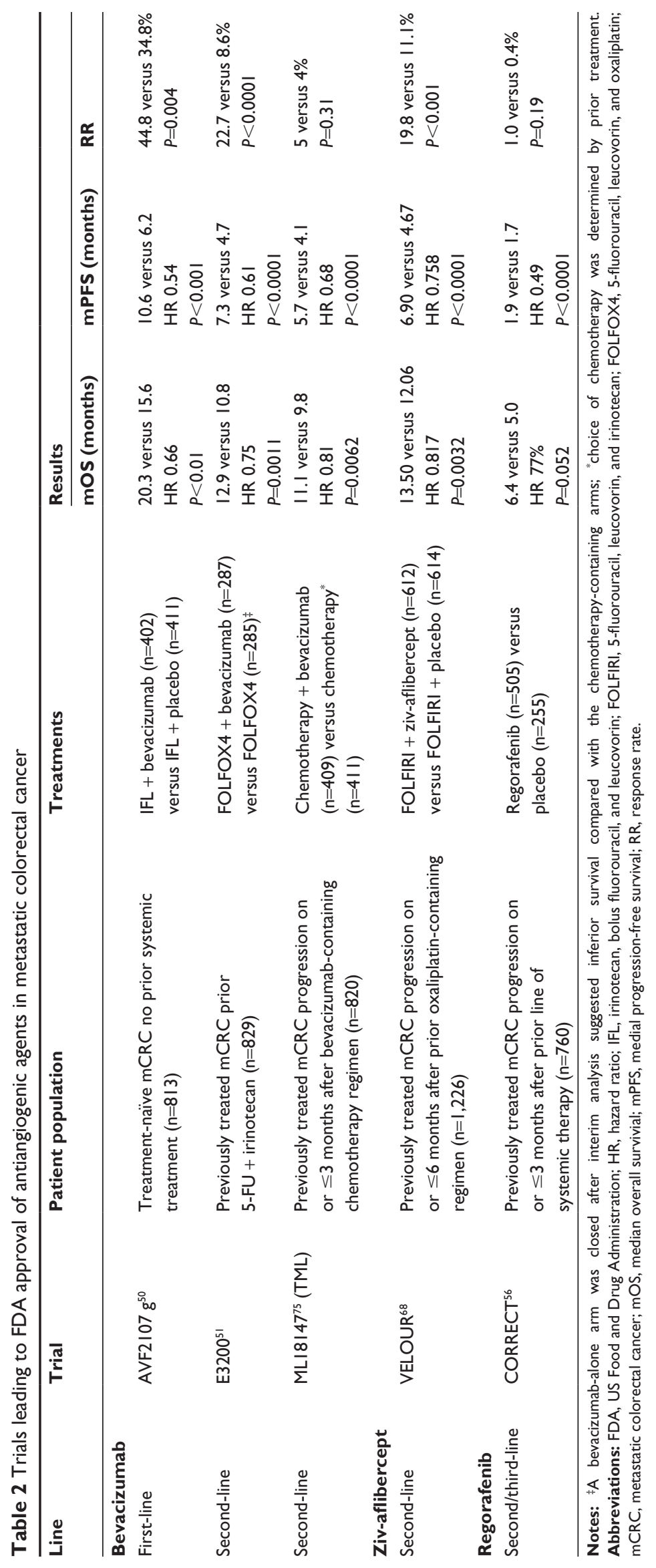


Table 3 Selected approved and active trials of ziv-aflibercept in colorectal cancer

\begin{tabular}{|c|c|c|}
\hline NCT number & Trial name & Phase \\
\hline NCT0I57I 284 & $\begin{array}{l}\text { Safety and quality of life study of aflibercept in patients with metastatic colorectal cancer previously } \\
\text { treated with an oxaliplatin-based regimen }\end{array}$ & III \\
\hline NCT0I646554 & $\begin{array}{l}\text { Efficacy of FOLFOX Versus FOLFOX plus aflibercept in K-ras mutant patients with resectable liver } \\
\text { metastases (BOS3) }\end{array}$ & II/III \\
\hline NCT01652196 & Aflibercept and FOLFOX6 treatment for previously untreated stage IV colorectal cancer & II \\
\hline NCT0I66I270 & $\begin{array}{l}\text { A study of aflibercept versus placebo with FOLFIRI in patients with metastatic colorectal cancer } \\
\text { previously treated with an oxaliplatin chemotherapy (AFLAME) }\end{array}$ & III \\
\hline NCT0I66I972 & Phase I/II study of capecitabine plus aflibercept to treat metastatic colorectal cancer (X-TRAP) & $\mathrm{I} / \mathrm{II}$ \\
\hline NCT0I669720 & Adjuvant aflibercept for metastatic colorectal cancer & $\|$ \\
\hline NCT0I802684 & OPTIMOX-aflibercept as first-line therapy in patients with unresectable metastatic colorectal cancer (VELVET) & II \\
\hline NCT0I882868 & $\begin{array}{l}\text { A study of aflibercept in combination with FOLFIRI in patients with second-line metastatic colorectal } \\
\text { cancer in Japan }\end{array}$ & II \\
\hline NCTOI889680 & Safety and effectiveness study of chemotherapy and ziv-aflibercept to treat metastatic colorectal cancer & II \\
\hline
\end{tabular}

Abbreviations: FOLFIRI, 5-fluorouracil, leucovorin, and irinotecan; FOLFOX4, 5-fluorouracil, leucovorin, and oxaliplatin; NCT, National Clinical Trials.

other pathways contributed to antiangiogenic resistance. In the absence of VEGF-A activity, binding of VEGF-C and VEGF-D to VEGFR-2 and VEGFR-3 has been shown to continue promotion of angiogenesis and tumor progression. ${ }^{82}$ As noted above, upregulation of VEGFR-3 by Notch has also been found to allow angiogenesis independent of the VEGF-A/VEGFR-2 pathway. ${ }^{44}$ Similarly, bFGF has been shown to act as a compensatory angiogenic factor following VEGF-A inhibition. ${ }^{83}$ The presence of these and other alternative pathways might necessitate the addition of multiple antiangiogenic agents. With the potent binding of VEGF-A and VEGFR-1 ligands provided by ziv-aflibercept, combination treatment targeting VEGFR-2 and VEGFR-3 might provide more complete coverage of the VEGF pathways. This might be accomplished through antibodies targeting the RTKs or their ligands, through tyrosine kinase inhibitors, which tend to have broader, more nonspecific activity, or novel direct angiogenic inhibitors. ${ }^{84}$ Numerous Phase I and Phase II studies have explored combination antiangiogenic regimens involving bevacizumab, ${ }^{85}$ some of which have shown increased antitumor activity. These studies have suffered from increased incidence and severity of adverse events.

\section{Predictive biomarkers}

A critical step needed for the utilization of ziv-aflibercept, and for antiangiogenic therapy in general, is the identification of patients more likely to derive a benefit from treatment. Preliminary results presented from a post hoc multivariate analysis of the VELOUR data identified a subset of patients with better outcomes in the ziv-aflibercept treatment arm. ${ }^{86}$ This subset was defined as patients with Eastern Cooperative Oncology Group (ECOG) performance status 0 or 1, with no more than one site of metastasis, and who had not experienced relapse during or within 6 months of completing oxaliplatinbased adjuvant therapy. In the ziv-aflibercept arm, 404 of 612 (66.0\%) patients met these criteria, as did 406 of 614 (66.1\%) in the placebo arm. Within this subset, mOS was more notably increased in the ziv-aflibercept arm compared to the placebo arm; 16.2 versus 13.1 months (adjusted HR 0.73; 95\% CI 0.61-0.86), respectively. mPFS also significantly improved; 7.2 versus 4.8 months (adjusted HR 0.68 ; 95\% CI $0.57-0.80$ ). ORR was $23.7 \%$ in the ziv-aflibercept arm, compared to $11 \%$ in the placebo arm. While this clinical classification may prove useful in selecting patients for ziv-aflibercept treatment, evaluation for associated predictive biomarkers would be far more valuable. As described above, measurement of the various angiogenic factors may provide some information regarding prognosis and response in CRC and other malignancies; ${ }^{87}$ however, no predictive biomarkers for antiangiogenic therapy have yet been identified. Some potential biomarkers are being studied for bevacizumab; ${ }^{88}$ similar exploration is needed for ziv-aflibercept. Such exploration would ideally include genetic and molecular profiling of tumors, immunohistochemistry, and serial evaluation of circulating levels. It would also include re-evaluation at the time of progression to evaluate potential resistance mechanism.

\section{Future directions}

Rather than solely seeking to provide an alternative or improvement to bevacizumab, investigation of ziv-aflibercept in settings where bevacizumab has yet to show a benefit may prove fruitful. These include upfront therapy with a combination of either FOLFIRI or FOLFOX, adjuvant therapy in earlier-stage $\mathrm{CRC},{ }^{89}$ and following liver metastectomy. ${ }^{90,91}$ Of patients with only liver metastases in VELOUR, a subgroup 
analysis of these patients revealed a significant improvement in OS and PFS, when compared to the remainder of the study population (HR 0.649 versus 0.868 ). Review of ongoing national clinical trials reveals active trials exploring some of these questions (Table 3 ).

\section{Conclusion}

The addition of anti-angiogenic agents has provided a significant, if limited, improvement in the treatment of mCRC. Ziv-aflibercept was approved for use in combination with FOLFIRI after demonstrating a survival benefit in patients that had progressed after an oxaliplatin-containing regimen. These results are important both in supplying another option in the treatment of $\mathrm{mCRC}$ and for validating another mechanism of blockade in tumor angiogenesis. In practice, however, the clinic application of ziv-aflibercept amongst other antiangiogenic agents has yet to be clarified. Predictive biomarkers are needed to help identify patients best suited for treatment with ziv-aflibercept. Research into the mechanisms of antiangiogenic research may also help guide the selection of ziv-aflibercept and the timing of its use.

\section{Disclosure}

The authors report no conflicts of interest in this work.

\section{References}

1. Ferlay J, Shin HR, Bray F, Forman D, Mathers C, Parkin DM. Estimates of worldwide burden of cancer in 2008: GLOBOCAN 2008. Int $J$ Cancer. 2010;127(12):2893-2917.

2. Bartlett DL, Chu E. Can metastatic colorectal cancer be cured? Oncology (Williston Park). 2012;26(3):266-275.

3. Gallagher DJ, Kemeny N. Metastatic colorectal cancer: from improved survival to potential cure. Oncology. 2010;78(3-4):237-248.

4. Carmeliet P, Jain RK. Angiogenesis in cancer and other diseases. Nature. 2000;407(6801):249-257.

5. Ferrara N, Gerber HP, LeCouter J. The biology of VEGF and its receptors. Nat Med. 2003;9(6):669-676.

6. Verheul HM, Voest EE, Schlingemann RO. Are tumours angiogenesisdependent? J Pathol. 2004;202(1):5-13.

7. Zetter BR. Angiogenesis and tumor metastasis. Annu Rev Med. 1998;49:407-424.

8. Folkman J. Anti-angiogenesis: new concept for therapy of solid tumors. Ann Surg. 1972;175(3):409-416.

9. Bergers G, Benjamin LE. Tumorigenesis and the angiogenic switch. Nat Rev Cancer. 2003;3(6):401-410.

10. Rafii S, Lyden D, Benezra R, Hattori K, Heissig B. Vascular and haematopoietic stem cells: novel targets for anti-angiogenesis therapy? Nat Rev Cancer. 2002;2(11):826-835.

11. Zadeh G, Koushan K, Baoping Q, Shannon P, Guha A. Role of angiopoietin-2 in regulating growth and vascularity of astrocytomas. J Oncol. 2010;2010:659231.

12. Weidner N. Intratumor microvessel density as a prognostic factor in cancer. Am J Pathol. 1995;147(1):9-19.

13. Catalano V, Turdo A, Di Franco S, Dieli F, Todaro M, Stassi G. Tumor and its microenvironment: A synergistic interplay. Semin Cancer Biol. 2013.
14. Ferrara N. Vascular endothelial growth factor: basic science and clinical progress. Endocr Rev. 2004;25(4):581-611.

15. Nagy JA, Vasile E, Feng D, et al. Vascular permeability factor/vascular endothelial growth factor induces lymphangiogenesis as well as angiogenesis. J Exp Med. 2002;196(11):1497-1506.

16. Kerbel RS. Tumor angiogenesis. N Engl J Med. 2008;358(19): 2039-2049.

17. Ferrara N. Vascular endothelial growth factor as a target for anticancer therapy. Oncologist. 2004;9 Suppl 1:2-10.

18. Hicklin DJ, Ellis LM. Role of the vascular endothelial growth factor pathway in tumor growth and angiogenesis. J Clin Oncol. 2005;23(5):1011-1027.

19. Shweiki D, Itin A, Soffer D, Keshet E. Vascular endothelial growth factor induced by hypoxia may mediate hypoxia-initiated angiogenesis. Nature. 1992;359(6398):843-845.

20. Sun W. Angiogenesis in metastatic colorectal cancer and the benefits of targeted therapy. J Hematol Oncol. 2012;5:63.

21. Kut C, Mac Gabhann F, Popel AS. Where is VEGF in the body? A meta-analysis of VEGF distribution in cancer. $\mathrm{Br} J$ Cancer. 2007;97(7):978-985.

22. Dvorak HF. Vascular permeability factor/vascular endothelial growth factor: a critical cytokine in tumor angiogenesis and a potential target for diagnosis and therapy. J Clin Oncol. 2002;20(21):4368-4380.

23. Shibuya M. Differential roles of vascular endothelial growth factor receptor-1 and receptor-2 in angiogenesis. J Biochem Mol Biol. 2006;39(5):469-478.

24. Shibuya M. Vascular endothelial growth factor receptor-1 (VEGFR-1/ Flt-1): a dual regulator for angiogenesis. Angiogenesis. 2006;9(4):225230; discussion 231.

25. Anisimov A, Leppänen VM, Tvorogov D, et al. The basis for the distinct biological activities of vascular endothelial growth factor receptor-1 ligands. Sci Signal. 2013;6(282):ra52.

26. Fischer C, Mazzone M, Jonckx B, Carmeliet P. FLT1 and its ligands VEGFB and PlGF: drug targets for anti-angiogenic therapy? Nat Rev Cancer. 2008;8(12):942-956.

27. Pavco PA, Bouhana KS, Gallegos AM, et al. Antitumor and antimetastatic activity of ribozymes targeting the messenger RNA of vascular endothelial growth factor receptors. Clin Cancer Res. 2000;6(5):2094-2103.

28. Hiratsuka S, Maru Y, Okada A, Seiki M, Noda T, Shibuya M. Involvement of Flt-1 tyrosine kinase (vascular endothelial growth factor receptor-1) in pathological angiogenesis. Cancer Res. 2001;61(3):1207-1213.

29. Carmeliet P, Moons L, Luttun A, et al. Synergism between vascular endothelial growth factor and placental growth factor contributes to angiogenesis and plasma extravasation in pathological conditions. Nat Med. 2001;7(5):575-583.

30. Xu L, Cochran DM, Tong RT, et al. Placenta growth factor overexpression inhibits tumor growth, angiogenesis, and metastasis by depleting vascular endothelial growth factor homodimers in orthotopic mouse models. Cancer Res. 2006;66(8):3971-3977.

31. Cao D, Hou M, Guan YS, Jiang M, Yang Y, Gou HF. Expression of HIF-1alpha and VEGF in colorectal cancer: association with clinical outcomes and prognostic implications. BMC Cancer. 2009; 9:432.

32. Yonekura H, Sakurai S, Liu X, et al. Placenta growth factor and vascular endothelial growth factor $\mathrm{B}$ and $\mathrm{C}$ expression in microvascular endothelial cells and pericytes. Implication in autocrine and paracrine regulation of angiogenesis. J Biol Chem. 1999;274(49): 35172-35178.

33. Nash AD, Baca M, Wright C, Scotney PD. The biology of vascular endothelial growth factor-B (VEGF-B). Pulm Pharmacol Ther. 2006;19(1):61-69.

34. Zhang F, Tang Z, Hou X, et al. VEGF-B is dispensable for blood vessel growth but critical for their survival, and VEGF-B targeting inh, ibits pathological angiogenesis. Proc Natl Acad Sci U S A. 2009;106(15):6152-6157. 
35. Jayasinghe C, Simiantonaki N, Kirkpatrick CJ. VEGF-B expression in colorectal carcinomas and its relevance for tumor progression. Histol Histopathol. 2013;28(5):647-653.

36. Jussila L, Alitalo K. Vascular growth factors and lymphangiogenesis. Physiol Rev. 2002;82(3):673-700.

37. Wang TB, Chen ZG, Wei XQ, Wei B, Dong WG. Serum vascular endothelial growth factor-C and lymphoangiogenesis are associated with the lymph node metastasis and prognosis of patients with colorectal cancer. ANZ J Surg. 2011;81(10):694-699.

38. Stacker SA, Achen MG. The VEGF signaling pathway in cancer: the road ahead. Chin J Cancer. 2013;32(6):297-302.

39. Su JL, Yen CJ, Chen PS, et al. The role of the VEGF-C/VEGFR-3 axis in cancer progression. Br J Cancer. 2007;96(4):541-545.

40. Martins SF, Garcia EA, Luz MA, Pardal F, Rodrigues M, Filho AL. Clinicopathological correlation and prognostic significance of VEGF-A, VEGF-C, VEGFR-2 and VEGFR-3 expression in colorectal cancer. Cancer Genomics Proteomics. 2013;10(2):55-67.

41. Huang H, Bhat A, Woodnutt G, Lappe R. Targeting the ANGPT-TIE2 pathway in malignancy. Nat Rev Cancer. 2010;10(8):575-585.

42. Qiao L, Wong BC. Role of Notch signaling in colorectal cancer. Carcinogenesis. 2009;30(12):1979-1986.

43. Jubb AM, Turley H, Moeller HC, et al. Expression of delta-like ligand 4 (D114) and markers of hypoxia in colon cancer. Br J Cancer. 2009;101(10):1749-1757.

44. Benedito R, Rocha SF, Woeste M, et al. Notch-dependent VEGFR3 upregulation allows angiogenesis without VEGF-VEGFR2 signalling. Nature. 2012;484(7392):110-114.

45. Hanrahan V, Currie MJ, Gunningham SP, et al. The angiogenic switch for vascular endothelial growth factor (VEGF)-A, VEGF-B, VEGF-C, and VEGF-D in the adenoma-carcinoma sequence during colorectal cancer progression. J Pathol. 2003;200(2):183-194.

46. Kang SM, Maeda K, Onoda N, et al. Combined analysis of p53 and vascular endothelial growth factor expression in colorectal carcinoma for determination of tumor vascularity and liver metastasis. Int J Cancer. 1997;74(5):502-507.

47. Rmali KA, Puntis MC, Jiang WG. Tumour-associated angiogenesis in human colorectal cancer. Colorectal Dis. 2007;9(1):3-14.

48. Saif MW. Anti-VEGF agents in metastatic colorectal cancer (mCRC): are they all alike? Cancer Manag Res. 2013;5:103-115.

49. Sung CY, Son MW, Ahn TS, Jung DJ, Lee MS, Baek MJ. Expression of placenta growth factor in colorectal carcinomas. J Korean Soc Coloproctol. 2012;28(6):315-320.

50. Hurwitz H, Fehrenbacher L, Novotny W, et al. Bevacizumab plus irinotecan, fluorouracil, and leucovorin for metastatic colorectal cancer. N Engl J Med. 2004;350(23):2335-2342.

51. Giantonio BJ, Catalano PJ, Meropol NJ, et al; Eastern Cooperative Oncology Group Study E3200. Bevacizumab in combination with oxaliplatin, fluorouracil, and leucovorin (FOLFOX4) for previously treated metastatic colorectal cancer: results from the Eastern Cooperative Oncology Group Study E3200. J Clin Oncol. 2007;25(12):1539-1544.

52. Chen HX, Cleck JN. Adverse effects of anticancer agents that target the VEGF pathway. Nat Rev Clin Oncol. 2009;6(8):465-477.

53. Nalluri SR, Chu D, Keresztes R, Zhu X, Wu S. Risk of venous thromboembolism with the angiogenesis inhibitor bevacizumab in cancer patients: a meta-analysis. JAMA. 2008;300(19):2277-2285.

54. Holash J, Davis S, Papadopoulos N, et al. VEGF-Trap: a VEGF blocker with potent antitumor effects. Proc Natl Acad Sci U S A. 2002;99(17):11393-11398.

55. Wilhelm SM, Dumas J, Adnane L, et al. Regorafenib (BAY 73-4506): a new oral multikinase inhibitor of angiogenic, stromal and oncogenic receptor tyrosine kinases with potent preclinical antitumor activity. Int J Cancer. 2011;129(1):245-255.

56. Grothey A, Van Cutsem E, Sobrero A, et al; CORRECT Study Group. Regorafenib monotherapy for previously treated metastatic colorectal cancer (CORRECT): an international, multicentre, randomised, placebocontrolled, phase 3 trial. Lancet. 2013;381(9863):303-312.
57. Rudge JS, Holash J, Hylton D, et al. VEGF Trap complex formation measures production rates of VEGF, providing a biomarker for predicting efficacious angiogenic blockade. Proc Natl Acad Sci U S A. 2007;104(47):18363-18370.

58. Papadopoulos N, Martin J, Ruan Q, et al. Binding and neutralization of vascular endothelial growth factor (VEGF) and related ligands by VEGF Trap, ranibizumab and bevacizumab. Angiogenesis. 2012;15(2): 171-185.

59. Gaya A, Tse V. A preclinical and clinical review of aflibercept for the management of cancer. Cancer Treat Rev. 2012;38(5):484-493.

60. Lockhart AC, Rothenberg ML, Dupont J, et al. Phase I study of intravenous vascular endothelial growth factor trap, aflibercept, in patients with advanced solid tumors. J Clin Oncol. 2010;28(2):207-214.

61. Yoshino T, Yamazaki K, Yamaguchi K, et al. A phase I study of intravenous aflibercept with FOLFIRI in Japanese patients with previously treated metastatic colorectal cancer. Invest New Drugs. 2013;31(4):910-917.

62. Van Cutsem E, Khayat D, Verslype C, et al. Phase I dose-escalation study of intravenous aflibercept administered in combination with irinotecan, 5-fluorouracil and leucovorin in patients with advanced solid tumours. Eur J Cancer. 2013;49(1):17-24.

63. Khayat D, Tejpar S, Spano JP, et al. Intravenous aflibercept administered in combination with irinotecan, 5-fluorouracil and leucovorin in patients with advanced solid tumours: results from the expansion cohort of a phase I study. Eur J Cancer. 2013;49(4):790-797.

64. Tang PA, Cohen SJ, Kollmannsberger C, et al. Phase II clinical and pharmacokinetic study of aflibercept in patients with previously treated metastatic colorectal cancer. Clin Cancer Res. 2012;18(21): 6023-6031.

65. Van Cutsem E, Peeters M, Siena S, et al. Open-label phase III trial of panitumumab plus best supportive care compared with best supportive care alone in patients with chemotherapy-refractory metastatic colorectal cancer. J Clin Oncol. 2007;25(13):1658-1664.

66. Karapetis CS, Khambata-Ford S, Jonker DJ, et al. K-ras mutations and benefit from cetuximab in advanced colorectal cancer. $N$ Engl J Med. 2008;359(17):1757-1765.

67. Pericay C, Folprecht G, Saunders M, et al. Phase 2 randomized, noncomparative, open-label study of aflibercept and modified FOLFOX6 in the first-line treatment of metastatic colorectal cancer (AFFIRM). Ann Oncol. 2012;23(Supplement 4):iv16.

68. Van Cutsem E, Tabernero J, Lakomy R, et al. Addition of aflibercept to fluorouracil, leucovorin, and irinotecan improves survival in a phase III randomized trial in patients with metastatic colorectal cancer previously treated with an oxaliplatin-based regimen. J Clin Oncol. 2012;30(28): 3499-3506.

69. Ruff P, Ferry D, Papamichael D, Lakomy R. O-0017 observed benefit of aflibercept in mCRC patients $\geq 65$ years old: results of a prespecified age-based analysis of the VELOUR study. Ann Oncol. 2013; 24(4):iv18.

70. Folkman J. Tumor angiogenesis: therapeutic implications. $N$ Engl J Med. 1971;285(21):1182-1186.

71. Chu E. An update on the current and emerging targeted agents in metastatic colorectal cancer. Clin Colorectal Cancer. 2012;11(1):1-13.

72. Troiani T, Martinelli E, Orditura M, De Vita F, Ciardiello F, Morgillo F. Beyond bevacizumab: new anti-VEGF strategies in colorectal cancer. Expert Opin Investig Drugs. 2012;21(7):949-959.

73. Petrelli F, Borgonovo K, Cabiddu M, et al. FOLFIRI-bevacizumab as first-line chemotherapy in 3500 patients with advanced colorectal cancer: a pooled analysis of 29 published trials. Clin Colorectal Cancer. 2013;12(3):145-151.

74. Saltz LB, Clarke S, Díaz-Rubio E, et al. Bevacizumab in combination with oxaliplatin-based chemotherapy as first-line therapy in metastatic colorectal cancer: a randomized phase III study. J Clin Oncol. 2008;26(12):2013-2019.

75. Bennouna J, Sastre J, Arnold D, et al; ML18147 Study Investigators. Continuation of bevacizumab after first progression in metastatic colorectal cancer (ML18147): a randomised phase 3 trial. Lancet Oncol. 2013;14(1):29-37. 
76. Grenon NN. Managing toxicities associated with antiangiogenic biologic agents in combination with chemotherapy for metastatic colorectal cancer. Clin J Oncol Nurs. 2013;17(4):425-433.

77. Morlock R, Yu E, Ray J. A cost-effectiveness analysis of bevacizumab (BV) plus chemotherapy (CT) versus aflibercept (AFLI) plus CT in patients with metastatic colorectal cancer $(\mathrm{mCRC})$ previously treated with BV. J Clin Oncol (Meeting Abstracts). J Clin Oncol. 2012;(34); abstr 417.

78. Kopetz S, Hoff PM, Morris JS, et al. Phase II trial of infusional fluorouracil, irinotecan, and bevacizumab for metastatic colorectal cancer: efficacy and circulating angiogenic biomarkers associated with therapeutic resistance. J Clin Oncol. 2010;28(3):453-459.

79. Willett CG, Duda DG, di Tomaso E, et al. Efficacy, safety, and biomarkers of neoadjuvant bevacizumab, radiation therapy, and fluorouracil in rectal cancer: a multidisciplinary phase II study. J Clin Oncol. 2009;27(18):3020-3026.

80. Loupakis F, Cremolini C, Fioravanti A, et al. Pharmacodynamic and pharmacogenetic angiogenesis-related markers of first-line FOLFOXIRI plus bevacizumab schedule in metastatic colorectal cancer. Br J Cancer. 2011;104(8):1262-1269.

81. Fischer C, Jonckx B, Mazzone M, et al. Anti-P1GF inhibits growth of $\operatorname{VEGF}(\mathrm{R})$-inhibitor-resistant tumors without affecting healthy vessels. Cell. 2007;131(3):463-475.

82. Clarke JM, Hurwitz HI. Understanding and targeting resistance to antiangiogenic therapies. J Gastrointest Oncol. 2013;4(3):253-263.

83. Lieu C, Heymach J, Overman M, Tran H, Kopetz S. Beyond VEGF: inhibition of the fibroblast growth factor pathway and antiangiogenesis. Clin Cancer Res. 2011;17(19):6130-6139.
84. Chen CT, Hung MC. Beyond anti-VEGF: dual-targeting antiangiogenic and antiproliferative therapy. Am J Transl Res. 2013;5(4): 393-403.

85. Moreno Garcia V, Basu B, Molife LR, Kaye SB. Combining antiangiogenics to overcome resistance: rationale and clinical experience. Clin Cancer Res. 2012;18(14):3750-3761.

86. Chau I, Joulain F, Iqbal S. A VELOUR post hoc subset analysis: prognostic groups and treatment outcomes in patients with metastatic colorectal cancer (mCRC) treated with aflibercept and FOLFIRI. Annal Oncol. 2013;24(suppl 4):iv91.

87. Wilson PM, LaBonte MJ, Lenz HJ. Assessing the in vivo efficacy of biologic antiangiogenic therapies. Cancer Chemother Pharmacol. 2013;71(1):1-12.

88. Lambrechts D, Lenz HJ, de Haas S, Carmeliet P, Scherer SJ. Markers of response for the antiangiogenic agent bevacizumab. J Clin Oncol. 2013;31(9):1219-1230.

89. Allegra CJ, Yothers G, O’Connell MJ, et al. Phase III trial assessing bevacizumab in stages II and III carcinoma of the colon: results of NSABP protocol C-08. J Clin Oncol. 2011;29(1):11-16.

90. Snoeren N, Voest EE, Bergman AM, et al. A randomized two arm phase III study in patients post radical resection of liver metastases of colorectal cancer to investigate bevacizumab in combination with capecitabine plus oxaliplatin (CAPOX) vs CAPOX alone as adjuvant treatment. BMC Cancer. 2010;10:545.

91. Kemeny NE, Jarnagin WR, Capanu M, et al. Randomized phase II trial of adjuvant hepatic arterial infusion and systemic chemotherapy with or without bevacizumab in patients with resected hepatic metastases from colorectal cancer. J Clin Oncol. 2011;29(7):884-889.
Biologics: Targets \& Therapy

\section{Publish your work in this journal}

Biologics: Targets \& Therapy is an international, peer-reviewed journal focusing on the patho-physiological rationale for and clinical application of Biologic agents in the management of autoimmune diseases, cancers or other pathologies where a molecular target can be identified. This journal is indexed on PubMed Central, CAS, EMBase, Scopus

\section{Dovepress}

and the Elsevier Bibliographic databases. The manuscript management system is completely online and includes a very quick and fair peerreview system, which is all easy to use. Visit http://www.dovepress. com/testimonials.php to read real quotes from published authors. 